\title{
Ionic Systems and Nanomaterials as Antiseptic and Disinfectant Agents for Surface Applications: A Review
}

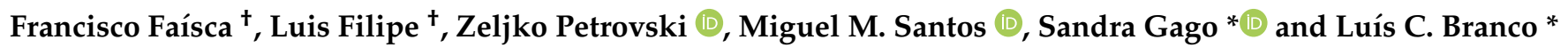 \\ LAQV, REQUIMTE, Departamento de Química da Faculdade de Ciências e Tecnologia, Universidade Nova de \\ Lisboa, 2829-516 Caparica, Portugal; f.faisca@campus.fct.unl.pt (F.F.); lm.filipe@campus.fct.unl.pt (L.F.); \\ z.petrovski@fct.unl.pt (Z.P.); miguelmsantos@fct.unl.pt (M.M.S.) \\ * Correspondence: s.gago@fct.unl.pt (S.G.); 1.branco@fct.unl.pt (L.C.B.) \\ + The first authorship is shared by two first authors.
}

Citation: Faísca, F.; Filipe, L.;

Petrovski, Z.; Santos, M.M.; Gago, S.;

Branco, L.C. Ionic Systems and

Nanomaterials as Antiseptic and Disinfectant Agents for Surface Applications: A Review. Surfaces 2021, 4,169-190. https://doi.org/ 10.3390 /surfaces4020016

Academic Editor: Gaetano Granozzi

Received: 15 March 2021

Accepted: 27 May 2021

Published: 7 June 2021

Publisher's Note: MDPI stays neutral with regard to jurisdictional claims in published maps and institutional affiliations.

Copyright: (c) 2021 by the authors. Licensee MDPI, Basel, Switzerland. This article is an open access article distributed under the terms and conditions of the Creative Commons Attribution (CC BY) license (https:// creativecommons.org/licenses/by/ $4.0 /)$.

\begin{abstract}
Antiseptics and disinfectants are extensively used for a variety of topical and hard-surface applications. A wide variety of biocides as active chemical agents is found in these products, including alcohols, phenols, iodine, and chlorine. Many of these active agents demonstrate broad-spectrum antimicrobial activity; however, the mode of action of these agents is not well-documented. This review is focused on several examples of ionic systems based on ionic surfactants and ionic liquids as well as nanomaterials and nanoparticles acting as antiseptics and disinfectants for surfaces. It is important to note that many of these biocides may be used singly or in combination in a variety of products, which vary considerably in activity against microorganisms. Antimicrobial activity can be influenced by several factors such as formulation effects, presence of an organic load, synergy, temperature, dilution, and test method. The most promissory compounds based on ionic systems and nanomaterials published in mainly the last decade is chronologically reported in this review.
\end{abstract}

Keywords: ionic liquids \& surfactants; surfaces; nanomaterials \& nanoparticles; antiseptic; disinfectant

\section{Introduction}

Antiseptics and disinfectants are used in different contexts with particular interests for hospitals and other health care places for several topical and hard-surface applications [1]. It is recognized that they are crucial for infection control strategies as well as the prevention of nosocomial infections. Taking into account the microbial contamination and infection risks for surfaces and food, among others, the development of new and more efficient antiseptics and disinfectants should be mandatory.

Various active chemical agents, named "biocides", are found in these products, and many of them are used for antisepsis, disinfection, and preservation.

"Biocide" is defined as a chemical agent that inactivates microorganisms. In general, the antiseptics are biocides or products that destroy or inhibit the growth of microorganisms on living tissue while disinfectants are similar but are generally products or biocides that are used on inanimate objects or surfaces.

The action mode of these active agents and the mechanisms of microbial resistance to antiseptics and disinfectants is an important parameter for researchers.

Considerable progress has been made in understanding the mechanisms of the antibacterial action of antiseptics and disinfectants. By contrast, studies on their modes of action against fungi viruses and protozoa is relatively limited.

The coronavirus disease (COVID-19) has spread throughout the world and was declared as a pandemic by the World Health Organization. This pandemic is caused by severe acute respiratory syndrome coronavirus-2 (SARS-CoV-2), and it has turned out to be one of the biggest threats of the century to human well-being. Epidemiological studies reported that human-human transmission through droplets is the principal route of transmission of 
SARS-CoV-2 [2]. One other possible route of transmission includes close contact of healthcare workers to SARS-CoV-2-contaminated surfaces in the healthcare environment. Both porous surfaces and nonporous surfaces (e.g., computers, sinks, and toilet seats) can be contaminated in healthcare facilities. Disinfection of healthcare surfaces is very important to prevent transmission of SARS-CoV-2 to healthcare workers and to other patients that occupy the facility. Several approaches can be used for disinfection including chemical disinfectants as well as enhanced coatings and surfaces with antimicrobial properties. Conventional chemical disinfectants often used in the healthcare facilities include sodium hypochlorite, potassium peroxymonosulfate, alcohols, quaternary ammonium compounds, and hydrogen peroxide, among others [3].

In this review, we will present an overview of antiseptics and disinfectants based on ionic systems such as ionic surfactants and ionic liquids as well as based on nanomaterials or nanoparticles and their further application in specific surfaces. We decided to organize this review considering the last decade of research and comparing two very promising approaches, ionic surfactants and ionic liquids (in comparison with conventional neutral or zwitterionic surfactants) and nanosystems including nanomaterials and metal nanoparticles as alternative anti-septic formulations. For both sub-topics of this review, the overview of antiseptics and disinfectants is reported in chronological data.

\section{Ionic Systems as Antiseptic and Disinfectants}

For the purposes of this review, "ionic systems" include two major families of compounds, ionic surfactants and ionic liquids acting as antiseptic or disinfectants to surfaces.

Surfactants are composed of hydrophilic and hydrophobic functionalities and they generally possess a propensity to adsorb at interfaces [4-7]. This class of surface-active compounds usually comprise alkyl chains responsible for hydrophobic interactions and a polar head that might be non-ionic, cationic, anionic, or zwitterionic, promoting the hydrophilic interactions. Cationic surfactants particularly are known as effective antimicrobials and antiseptics [7].

Ionic liquids (ILs) are composed of organic cations and anions possessing a low melting point (below $100{ }^{\circ} \mathrm{C}$ ), and despite being first synthesized in 1914, have only found exponential scientific interest in past two decades. Since then, ILs showed peculiar physicalchemical properties such as reduced vapor pressure, non-flammability, high chemical and thermal stability, and the possibility to tune their properties according to suitable cationanion combinations [8].

It is possible to consider a large number of IL applications $[9,10]$ for different research areas such as polymer science, analytical chemistry, organic synthetic reactions, (bio)catalytic reactions, $\mathrm{CO}_{2}$ capture and valorization, biomass processing, and pharmaceutical chemistry, amongst others.

Many ILs with antimicrobial properties that have been investigated fall under the family of Quaternary Ammonium Salts (QAS), widely used antimicrobials, and cationic surfactants, due to the structural similarities with ILs, that is their ionic nature and proven microbial activity $[9,11,12]$. It was found that compounds with longer alkyl chains on the cations were exponentially more active than their smaller alkyl chain counter parts, which had the added benefit of potentiating the compounds surface activity.

In fact, it was noted that aggregation behavior, critical micellar concentration (CMC), and efficiency of surface tension reduction ( $\mathrm{pC}$ 20) parameters of surface activity had a strong correlation with biological activity and were likely closely related to the mechanism of action of these compounds [9,13-17]. Still, it is important to point out that because of the inherent variety to ILs, these observations are not clearly correlated with other kinds of antimicrobial ILs.

Despite the vastness of families of organic compounds possessing antimicrobial activities, in this review, we will focus on studies that directly or indirectly investigated the development of ionic systems (ILs and ionic surfactants) with surface activity or application on surfaces, as well as antimicrobial or antiseptic potential. 
Starting in 2004, Demberelnyamba et al. [18] investigated the potential of imidazolium and pyrrolidinonium salts in the development of disinfectants and antiseptics. In this work, they developed three series of ILs based on 1-alkyl-3-methylimidazolium, 1-alkyl-3hydroxyethyl-2-methylimidazolium, and N-dodecyl-N-hydroxyethylpyrrolidinonium (see Figure 1). These cations containing large alkyl chains from $C_{8}$ to $C_{16}$ were combined with chloride and bromide anions. All prepared compounds showed a low minimum inhibitory concentrations, in particular in the cases of imidazolium-based ILs with very good activity against Gram-positive and Gram-negative bacteria.

It is considered that the major factors in inhibitory response are the structure of functional groups and the length of alkyl chain on the cation. In the reported study, the authors concluded there was a close interaction between surface activity and biological activity since the imidazolium and pyrrolidinonium salts synthesized are highly surfaceactive agents. In this context, their antimicrobial activities were enhanced by increased amphiphilicity of the chosen surfactants.
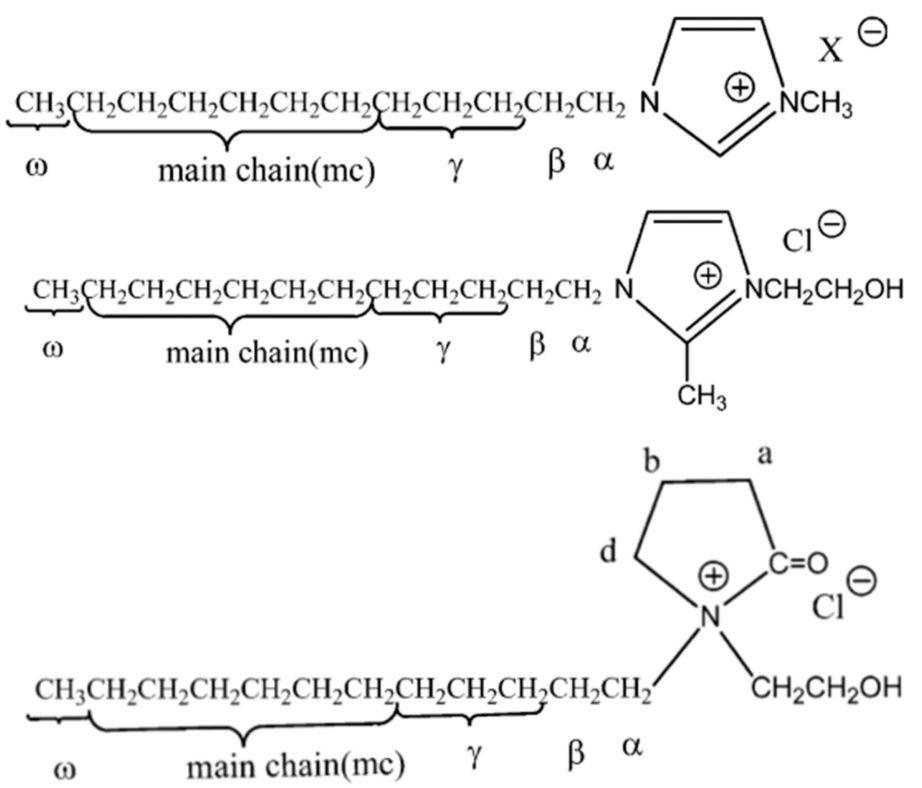

Figure 1. The structures of 1-alkyl-3-methylimidazolium, 1-alkyl-3-hydroxyethyl-2-methylimidazolium and N-dodecyl-N-hydroxyethylpyrrolidinonium ILs, respectively. Adapted from [18].

Pernak et al. [19] reported the antimicrobial potential of 1-[(1R,2S,5R)-(-)-menthoxymethyl]3-alkylimidazolium ILs. The salts demonstrated high antimicrobial activity, particularly in those with longer alkyl chains, regarding those with at least five carbons as active, and those higher than eight carbons as superactive, represented in Figure 2, reaching stronger biocidal activity compared to the control antibiotic benzalkonium chloride. Furthermore, 1-[(1R,2S,5R)-(-)-menthoxymethyl]-3-alkylimidazolium chlorides proved to have an excellent anti-electrostatic effect, though this could be inhibited by other substituents such as methyl groups or the presence of aromatic rings, which was attributed to their impact on aggregation. These properties led the researchers to be confident in the application of these ILs as disinfectants with anti-electrostatic activity. 

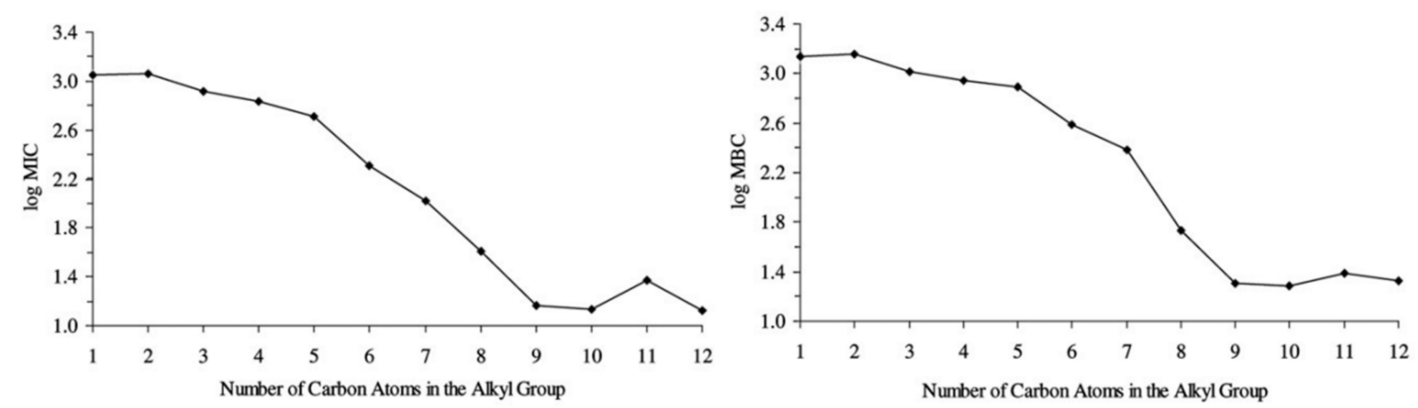

Figure 2. Mean Minimum Inhibitory Concentrations (MIC) and Minimum Biocidal Concentrations (MBC) for menthoxymethyl]-3-alkylimidazolium ILs in relation to the number of carbon atoms in the alkyl chain. Adapted from [19].

Foksowicz-Flaczyk et al. [20] tested the efficacy of ecofriendly antimicrobial ionic liquid didecyldimethylammonium nitrate, a quaternary ammonium salt, in the finishing lignocellulosic textiles to protect against microbial degradation. They found that $8 \mu \mathrm{g}$ on $1 \mathrm{~g}$ of textile was effective against several Gram-positive and Gram-negative bacteria, with a distinct inhibition zone. The authors concluded that it represented a promising compound to replace non-environmentally safe compounds.

Like previous work published by Pernak [19], the methylimidazolium cations were synthesized with a larger alkyl chain with similar conclusions: Longer chains were paramount to higher anti microbials activities, while the choice of counter ions only had a small impact. However, they also noted that the correlation between the alkyl chain and biological activity was only true up to a cutoff point of 16 to 18 carbons.

Additionally, aqueous solutions of 1-alkyl-3-methylimidazolium with longer alkyl chains, between $C_{8}$ and $C_{18}$, act as a surfactant and demonstrated the ability to selfassemble into micellar aggregates, which raises their efficiency of tension reduction, pC20, meaning that longer alkyl chains lead to higher surface and biological activity, indicating a correlation between both.

In 2011, Cybulski et al. [21] evaluated the effectiveness of quaternary ammonium salts, didecyldimethylammonium, benzalkonium with alkyl groups, and domiphen [N,Ndimethyl-N-(2-phenoxyethyl)-1-dode-canaminium], as illustrated in Figure 3, with the R and $\mathrm{L}$ enantiomers of mandelate and L-prolinate anions detailed in Figure 4. These proved to be effective anti-microbial agents, particularly didecyldimethylammonium that, with two long alkyl chains, had both the most effective antimicrobial activity and impact on $\mathrm{CMC}$ values regardless of counter-ions, which proved to be much less impactful than the choice of cations. Finally, even though the ILs were synthesized specifically as chiral ILs, the team found no relevant correlation between quirality and antimicrobial activity, only showing relevance for phytotoxicity values.

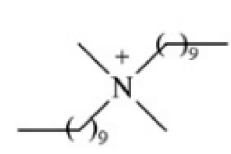

[DDA]

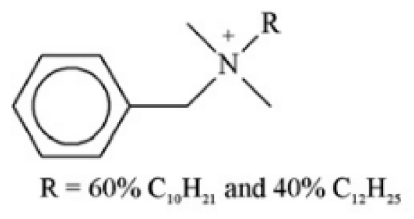

[BA]

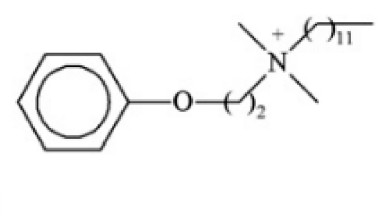

[DOM]

Figure 3. Structures of the cations including didecyldimethylammonium DDA, benzalkonium BA, and domiphen [N,N-dimethyl-N-(2-phenoxyethyl)-1-dodecanaminium] [DOM]. Adapted from [21]. 
<smiles>O=C(O)C(O)c1ccccc1</smiles>

MAN<smiles>O=C([O-])[C@@H](O)c1ccccc1</smiles>

$R$-MAN<smiles>O=C([O-])[C@@H](O)c1ccccc1</smiles>

$S$-MAN<smiles>O=C([O-])[C@H]1CCCN1</smiles>

L-PRO

Figure 4. Structures of the anions including racemic mandelate [MAN], R-mandelate [R-MAN], L-mandelate[L-MAN], and L-proline [L-PRO]. Adapted from [21].

Then, Yagci et al. [22] developed polyurethane coatings with incorporated quaternary ammonium salt-based ILs with chloride and sulfoxide counter anions. These components were incorporated with spacers of different lengths, and the longest showed the most effective antimicrobial activity against Gram-positive Staphylococcus aureus and Gramnegative Escherichia coli. For other side, the shorter was only effective against Gramnegative E. coli. suggesting that the action mode can involve the contact killing instead of leaching of biocide material. Through water contact angle measurements, it was determined that increased concentrations of ILs result in more hydrophilic surfaces and increased water sensitivity inducing the water swelling.

Venkata et al. [23] also investigated two ILs, 1-dodecyl-3-methylimidazolium iodide and cetyl-trimethylammonium bromide, as antimicrobial and antibiofilm agents for industrial and medical applications. In particular, the IL 1-dodecyl-3-methylimidazolium iodide demonstrated potent anti- microbial and anti-biofilm activity against Gram-positive S. aureus, and to a minor degree, against Gram-negative P. aeruginosa. Furthermore, it was noted that this IL had comparable anti-biofilm activity to the well-known cationic surfactant CTAB (see Figure 5) impairing growth, development, and attachment on polystyrene surfaces exposed to fresh water. It was found that the ionic liquid increased the cell membrane permeability for both Gram-positive and Gram-negative bacteria possessing promissory applications in the control of contamination and infection.

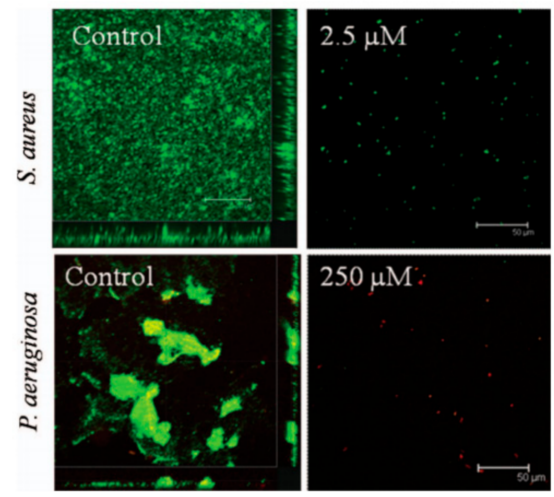

Figure 5. Biofilm formation by S. aureus and P. aeruginosa PAO1 in TSB medium (control) and in the presence of $\left[\mathrm{C}_{12} \mathrm{MIM}\right] \mathrm{I}$. S. aureus were stained with acridine orange and imaged using confocal microscopy. P. aeruginosa biofilms were stained with BacLight live/dead bacterial viability staining. Green $\frac{1}{4}$ SYTO 9; red $\frac{1}{4}$ propidium iodide. Adapted from [23].

In 2015, El-Shamy et al. [24] investigated the antibacterial effects of a pyrrolinidiniumbased ionic liquid ([Py1,4][TfO]), against planktonic and sessile bacteria on mild steel surfaces. They found that this IL adsorb onto the steel surface and formed a protective film that would inhibit the adhesion of bacteria and the subsequent accumulation of biofilms and protected against the corrosive attack as well as effectively inhibit bacterial growth.

Inhibitory effects of corrosion of mild steel were observed with concentrations of $100 \mathrm{ppm}$ and with an efficiency in the excess of $80 \%$ in solutions of $3.5 \% \mathrm{NaCl}$, and antibacterial effects were 2 to 3 times more effective against sessile bacteria than planktonic bacteria. 
In 2017, Singh et al. [25] investigated the surface properties of lauryl alcohol-based gemini surfactants (BSGSLA's) and acetyl alcohol-based gemini surfactants (BSGSCA's), when combined with organic salts, sodium salicylate and sodium benzoate, and inorganic salts, sodium chloride, and potassium chloride as indicated in Figure 6.

The authors reported that introducing the salts in higher concentrations improved the surface activity of the tested surfactants. In general, the longer alkyl chain of BSGSCA's is more favorable in terms of CMCs and emulsion stabilities, while the lowest alky chain of BSGSLA's promotes the foam stability. They also found that the choice of salts had a significant impact on performance with the general trend of performance of the organic salts being the most beneficial and sodium salicylate being the overall best option. Furthermore, other surfactant modifications were also found to be beneficial when in combination with the salts, such as an elongated spacer group and enlarged alkyl chains.

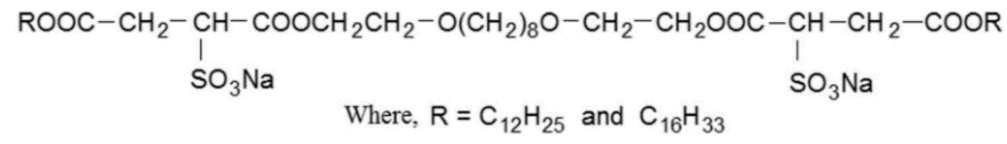

(1) Bis-sulfosuccinate anionic gemini surfactants<smiles></smiles>

(2) Sodium Salicylate (NaSal)

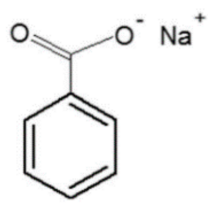

(3) Sodium benzoate (NaBenz)

Figure 6. Molecular structures of (1) bis-sulfosuccinate anionic gemini surfactants, (2) sodium salicylate (NaSal), and (3) sodium benzoate (NaBenz). Adapted from [25].

Marek et al. [26] synthesized three series of pyridine-based cationic surfactants 2-, 3-, and 4- hydroxymethylpyridinium with elongating alkyl chains ranging from $\mathrm{C} 10$ to $\mathrm{C} 18$, as alternatives to the widely used quaternary ammonium salts, and then they evaluated their antimicrobial and CMCs. The most effective anti-microbial activity and highest CMC values were found on the surfactants with the longest alkyl chains, and the ILs were, in general, more potent on Gram-positive bacteria but also effective on Gram-negative bacteria. It is also mentioned that the position of the hydroxymethyl group on the pyridinium cation was insignificant for antimicrobial or surface activity.

Ossowicz et al. [27] explored the potential of benzalkonium quaternary ammonium salts combined with amino acids as anions (see Figure 7) as a non-toxic alternative to counter ions. The antimicrobial and surface activity of each compound and a comparison with benzalkonium chloride was performed. In general, they found a similar surface activity for new salts and previous ones, but a higher antimicrobial activity against Grampositive bacteria of the Staphylococcus genus. In the case of Gram-negative Escherichia and Raoultella genus, salts containing amino acid anions showed lower activity on microorganisms, being 2 to 10 times less active than the antimicrobial activity of benzalkonium chloride.
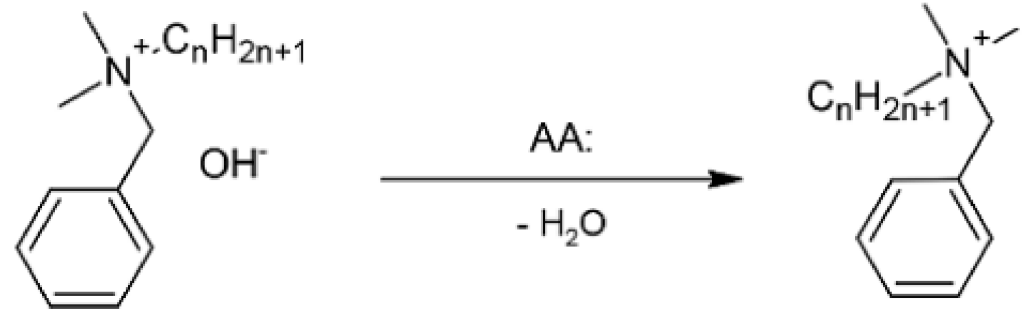

$\mathrm{AA}^{-}$

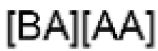

Figure 7. Schematic preparation of benzalkonium [BA] amino acid [AA] salts. Adapted from [27]. 
In 2019, Forero-Doria et al. [28] investigated the potential of imidazole based ILs with p-coumaric and cinnamic acid as alternative strategies to tackle bacterial resistance to antibiotics. Researchers synthesized N-alkylimidazolium salts in an elongating alkyl chain and tested antimicrobial and antibiofilm activity against Gram-positive and Gram-negative bacteria. As of previous studies, a correlation was observed between antimicrobial activity between chain length and antibacterial effectiveness, with longer chains ranging from C8 to C10 having a more potent effect, and with the salts derived from p-coumaric acid having a higher efficacy, particularly against Gram-positive bacteria. Salts synthesized from cinnamic acid showed better inhibition of the formation of biofilm in the case of S. aureus for $52 \%$ inhibition. The team concluded that salts derived from $\mathrm{p}$-coumaric acid could be used as antibacterial compounds while p-cinnamic acid could be used as antibiofilm compounds.

Rzemieniecki et al. [29] synthesized 1-alkyl-4-hydroxy-1-methylpiperidinium ILs combined with mandelic acid (S, R enantiomers or racemic) as well as with an elongating alkyl chain, resulting in salts with a heterocyclic cation with cis and trans isomeric forms. They already reported that the increasing of the alky chain resulted in a linear increase in surface activity parameters, pC20, as well as the antimicrobial activity against a series of Gram-positive and Gram-negative bacteria (see Figure 8). They also indicated no significant influence related to chiral form of the anion, but a strong correlation between antimicrobial activity and the amphiphilicity of the ionic liquid.
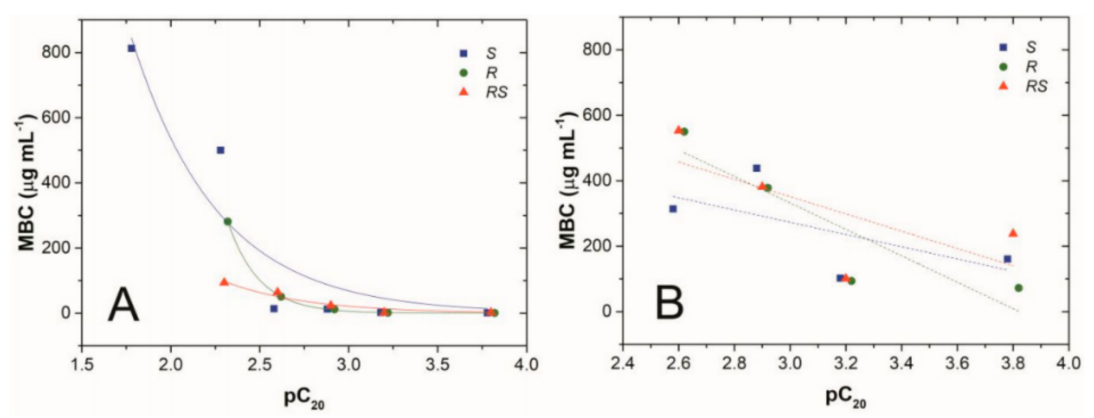

Figure 8. Relationships between adsorption efficiency (pC20) and mean values of MBC towards Gram-positive bacteria (A), Gram-negative bacteria (B) with (S)-mandelate, (R)-mandelate, and (RS)-mandelate anions [29].

In 2020, Anandkumar et al. [30] tested the potential of ILs against biofilm formation on materials used in cooling water systems. The authors synthesized two imidazolium-based ILs (1-ethyl 3-methylimidazolium tetrafluoroborate and 1-butyl-3-methylimidazolium chloride) and one piperidinium-based IL (N-methyl-N-propylpiperidinium bis(trifluoromethylsulfonyl)imide) and then they tested all of them in terms of efficacy against cultures of planktonic and sessile on titanium and carbon steel surfaces. They found that 25ppm of N-methyl-Npropylpiperidinium bis(trifluoromethylsulfonyl)imide and 1-Ethyl 3-Methylimidazolium tetrafluoroborate ILs provided anti-bacterial activity against planktonic bacteria while concentrations of 50ppm resulted in 80 to $90 \%$ biofilm inhibition and $90 \%$ eradication. In general, the IL 1-ethyl 3-methylimidazolium tetrafluoroborate also proved to be the most efficient anti-adhesive ionic liquid on sessile bacteria.

Most of the work presented in this section stems from experiments on already wellknown compounds with antiseptic activity, namely quaternary ammonium salts and gemini surfactants, and the added influence of ionic liquids or organic salts instead of the development of novel compounds based on ionic liquids. To this effect, most researchers found that the introduction of conventional compounds as ionic systems has a positive effect on antimicrobial activity. Most researchers found that there was a significant correlation between alkyl chain lengths and biocidal activity.

However, research regarding more broad biocidal ionic liquids have found that activity is correlated with higher molecular weights in general, not necessarily just alkyl chains [31]. 
On the other hand, these surface-active antiseptics are based on amphiphilic molecules whose alkyl chains are a main factor in their lipophilicity, thus could be more relevant for these particular molecules.

\section{Nanomaterials and Nanoparticles as Antiseptic and Disinfectants}

There is a constant demand for novel products and materials that can suppress the action of bacteria, fungi, and the respective formation of biofilms on top of surfaces. In such a context, it is of paramount importance to understand the recent efforts that scientific research has made to approach this problem. Of particular interest lies the applications of nanoscopic materials in the disinfection of surfaces and prevention of contamination.

Nanomaterials are, by definition, materials with sizes, diameters, and widths in the nanometric region. The development of nanomaterials has seen an increase in later years, due to the advancements made in such an area. More than ever, it is possible to synthetize nanomaterials with a precise control over important parameters such as the growth of the material and its topology. Nanomaterials come in a wide range of possible forms, as they enclose a broad class of materials. Given that, nanomaterials can comprise nanoparticles, usually globular aggregates of either organic or inorganic material with well-defined sizes and porosity; nanocomposites, which are materials that are comprised of more than one component, but always with nano-sizes; or other heterogenous materials such as polymers or dendrimers.

Nanomaterials are tunable, stable, and non-volatile under most conditions, which makes them suitable candidates for the development of novel systems for controlling and preventing bacterial adhesion on surfaces. Due to their inherent characteristics, it is possible to adapt the material for several different conditions, including the loading of biocides in the core, the functionalization of the surface of the material, the development of responsive materials, or even the direct incorporation of the materials in matrixes. This review gives particular emphasis to the action of different metal ions-especially silver-against living organisms, as they have been extensively studied and reported. Most nanomaterials reported here can be prepared through facile routes, with no timeconsuming methodologies and through environmentally friendly techniques. Furthermore, some of the nanomaterials could be stored for prolonged periods (in some cases, more than one year, while retaining the antibacterial properties) and are also reusable or regenerable. Nonetheless, one of the most remarkable aspect of such nanomaterials is their outstanding capability to deactivate biofilms and kill bacteria. Because they can, in most cases, be selectively loaded with a particular concentration of biocide, are stable, and bacteria have no innate way of resisting nanomaterials, the area of nano science can be prone to the development of many different disinfection strategies.

Therefore, nanomaterials that have already been used and tested before against bacteria are reported here. The following paragraphs describe representative reports found in the scientific literature that covers the study and implementation of nanomaterial systems against several strains of bacteria, fungi, or biofilms. The articles are presented in chronological order, starting from the eldest and moving to the more recent ones.

In 2007, Sawada et al. [32] prepared nanocomposites consisting of fluoroalkyl endcapped oligomers with encapsulated biocides in a silica matrix. The synthetic methodology described is rather simple as it only depends on the hybridization of a homogenous methanolic solution containing the fluoroalkyl moieties, the biocides, and source of silica, such as TEOS. N-(1,1-dimethyl-3-oxobutyl)acrylamide (DOBAA), N,N-dimethylacrylamide (DMAA), and acrylic acid (ACA) were the fluorinated oligomers used in this study. Hibitane, hinokitiol, and hinokioil were the biocides used to encapsulate inside the material. The fluorinated nanocomposites obtained were nanometer-size-controlled between 86 and $563 \mathrm{~nm}$ and presented a good dispersibility and stability in methanol and water. The authors tested the various products against strains of Staphyloccocus Aureus, and the results showed that all the three biocides exhibited excellent results with the increasing of its antibacterial activity for higher amounts of encapsulated biocides. Extremely higher 
antibacterial activity was obtained for hinokitiol content above $13.6 \%$ and hinokioil above $23.3 \%$, as compared to the biocides solely dispersed in solution. These nanocomposites were also applied for the surface modification of poly(methyl methacrylate) (PMMA).

In 2010, Ghosh et al. [33] studied the influence of treating textile fabric with modified dendrimers to increase the antibacterial activity of the material. The primary amine end groups of poly(amidoamine) (PAMAM) G-3 dendrimer were converted into ammonium functionalities. The authors took advantage of the unique properties of dendrimers-such as the globular shaping and the ability to tune, edit, and modify the cavities-to use them as a template for the synthesis of silver nanoparticles and prepared the complexes $\mathrm{AgNO}_{3}-\mathrm{PAMAM}$ (G3) and MesoSilver-PAMAM (G3). The two classes of nanocomposites obtained were soaked in nylon and cotton textile fabrics and assayed for their antibacterial profile. All the treated fabrics exhibited higher antibacterial activities, when compared to the untreated material, as they were visually able to prevent the growth and proliferation of bacteria. The ammonium-treated textiles showed the most satisfactory results, as a full $12 \mathrm{~mm}$ inhibition area was found in the fabric, in which no bacteria could develop. As for the silver nanocomposites, whilst being active against bacteria, they only created an inhibition zone of approximately $2.6 \mathrm{~mm}$. Nonetheless, the results are promising and lay the ground for an easy application in the treatment of textile fibers.

Chapman et al. [34] addressed the biofouling distress by adapting various period-four metals as the active components to dope sol-gel (SG) coatings with antibacterial activity. The metallic nanoparticles (MNPs) were obtained through a poly-ol reduction method, which involves the use of a reducing reagent (in this case the agent used was $\mathrm{NaBH}_{4}$ ) in a solution of ethane-1,2-diol, as can be observed in Figure 9. To that solution, metal salts are added, which suffer reduction from the medium and engage in nanoparticle formation. The metals used in this studied were the chloride salts of Scandium (III), Titanium (III), Vanadium (II), Manganese (II), Iron (III), Cobalt (II), Nickel (II), Copper (II), Zinc (II), Germanium (II), Selenium (IV), and the nitrate salts of Chromium (III) and Gallium (III), which gave rise to the corresponding metal nanoparticles, all below $90 \mathrm{~nm}$ of diameter.

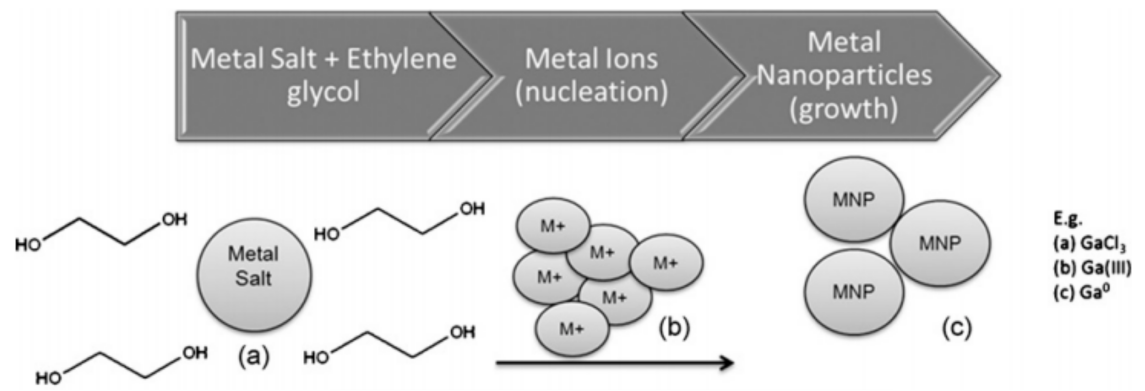

Figure 9. Schematic representation of the method employed by Regan's team. Adapted from [33].

The MNPs-doped sol-gels were tested in environmental freshwater for three whole days and the bacterial adhesion was counted as well as the minimum inhibitory concentration needed to inhibit bacterial growth. The results suggested that $\mathrm{Ga}, \mathrm{Ge}$, and Se nanoparticles showed promising levels of biomass inhibition with Ga showing up to 125\% better biomass resistance compared to the sol-gel blank. GaMNPs-doped sol-gels gave better biofouling resistance to microorganisms (500\% increase) compared to any of the other MNP-doped sol-gels, while $\mathrm{Cr}, \mathrm{Mn}, \mathrm{Ge}$, and Se all gave similar results and proved to be effective at resisting microbial attachment (up to 200\%). However, even though some MNPs showed superior antifouling response to others, all the tested materials still outperform, in some cases by 150\%, the sol-gel undoped control.

In 2012, Miranda et al. [35] proposed a protocol for the synthesis of hydroxyapatite/silver nanocomposites for the final purpose of controlling the proliferation of bacteria in ceramic and bone-like materials in medical uses. The hydroxyapatite matrix was synthetized according to the sol-gel method followed by subsequent deposition of the silver ions, added in the form of $\mathrm{AgNO}_{3}$. For this latter step of silver deposition, two different 
routes were studied: A thermal (the solution was heated at $350{ }^{\circ} \mathrm{C}$ ) and a chemical one (using a reducing agent, like $\mathrm{NaBH}_{4}$ ). Both methods produced similar products, which were observed to possess a good dispersibility of silver ions. However, in the thermal treatment, the formed nanoparticles were bigger than the corresponding chemically obtained nanoparticles. Because the surface area exposed to the microorganisms tends to increase with the decrease of the diameter of the nanoparticles, the chemically reduced nanoparticles were expected to exceed the thermally reduced ones in the antibacterial activity. In fact, the results showed that the chemically reduced nanoparticles exhibited relatively higher antibacterial activities than the corresponding thermally reduced ones. However, in the case of yeast, only the chemical-reduced nanoparticles were able to produce any significant effect, above the commercial antimicrobial threshold. Besides the antibacterial tests, the authors also analyzed the lixiviated residues for the quantitative analysis of the content in silver and to deliver a preliminary toxicity assay, as it is depicted in Table 1 . The results allowed to conclude that in each case, the concentration of silver was below the accepted toxicity level for silver ions. With this work, the authors proposed that the combination of the bioactivity of the ceramic matrix with the biocide activity of the silver nanoparticles makes this material an excellent candidate for implants, bone filling, and reconstructive surgery applications.

Table 1. Concentration of silver ions in lixiviated residues of the antimicrobial tests, with respective $\mathrm{pH}$ values. Adapted from [35].

\begin{tabular}{ccccc}
\hline \multirow{2}{*}{ Bacteria } & \multicolumn{2}{c}{ Thermally Reduced AgNPs } & \multicolumn{2}{c}{ Chemically Reduced AgNPs } \\
\cline { 2 - 5 } & Ag Concentration (in ppm) & $\mathbf{p H}$ & Ag Concentration (in ppm) & $\mathbf{p H}$ \\
\hline E. Coli & 1.981 & 6.716 & 1.184 & 6.57 \\
\hline M. Luteus & 1.199 & 7.030 & 1.342 & 6.93 \\
\hline I. orientalis & 0.008 & 6.733 & 1.614 & 6.64 \\
\hline
\end{tabular}

The presence of ions in aqueous systems has the unwanted ability to oxidize silver ions, rendering them ineffective against bacterial infections, which makes the application of silver nanoparticles for biofilm formation prevention difficult. To overcome this issue, in 2013, Das et al. devised an alternative way to incorporate silver ions in a silica matrix to overcome such issues [36]. A nano-silica-silver nanoparticles composite (NSAgNPs) were obtained through a protein-mediated reduction of silver ions, with nano-silica powder and a protein extract from R. Oryzae. The obtained NSAgNPs presented high stability and were able to protect the silver ions from undesired oxidation. The composite was tested for its ability to remove several types of ionic paints and nearly $99 \%$ of all tested dyes were removed by this system under $30 \mathrm{~min}$. The antibacterial properties were also evaluated, and the results showed prolonged antibacterial activity on planktonic cells and biofilms of Escherichia coli and Pseudomonas aeruginosa. NSAgNPs was able to avoid the initial attachment of bacteria and therefore improved the antifouling properties of material, which prevented any biofilm formation. The authors also proposed that the immobilization of protein-coated AgNPs significantly improved the antibacterial property of NSAgNP, as they outperformed the mere AgNPs in the antibacterial tests. Moreover, the efficient dye adsorption, reusability, and antifouling properties of this composite can open new opportunities for new cost-effective and ecofriendly methods for water purification.

Liu et al. [37] prepared a set of silver-nanoparticles for application on textile fabrics. The novelty of this work lies in a one-pot and in situ method to directly assemble the nanoparticles in the cotton fibers by their surface modification with 3-aminopropyltriethoxysilane. This modification converts the terminal positions of the cotton polysaccharides in amine groups, which can adsorb the silver ions and slowly release it upon contact with bacteria, as can be seen in Figure 10. By this way, the leaching of silver ions into water effluents is overcome. The antibacterial tests were satisfactory, as more than $99 \%$ of the bacteria growth 
ceased within one hour of contact with the material. Additionally, the textiles have also demonstrated continued anti-bacterial efficiency after several cycles of washing, which might help establish this method for the application in the continuous disinfection of textile fabrics in industrial proportions.

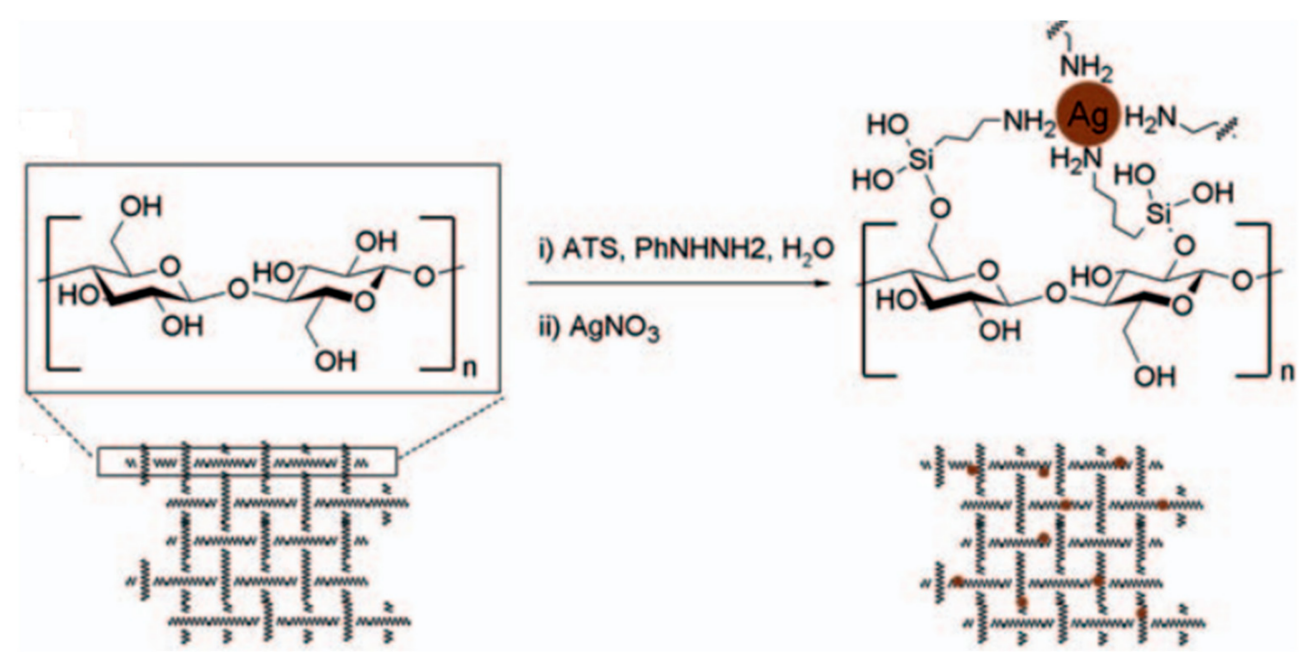

Figure 10. Schematic representation of the formation of silver nanoparticles directly in the cotton fibers. Retrieved from [37].

Zheng et al. [38] used mesoporous silica nanoparticles (MSNs) as a vehicle to deliver self-healing coating for the prevention of biofilm formation. In this study, a $\mathrm{pH} /$ sulfide ion responsive release system was developed based on MSNs, forming a copper benzotriazole (Cu-BTA) complex at the openings of the mesopores into which benzotriazole (BTA, a corrosion inhibitor) and benzalkonium chloride (BCA, a biocide for the antibacterial effect) were loaded. The preparation starts with the functionalization of the pore openings of MSNs with one of the three different aliphatic amines, aminopropyltriethoxysilane, N-(3trimethoxysilylpropyl)ethylenediamine, and N-(3-trimethoxysilylpropyl)diethylenetriamine, before loading with the active species. Among these functionalized MSNs, the one with diamine showed the highest loading capacity and ideal release profile. Through this strategy, the spontaneous leakage and premature release of active species are avoided, and a responsive release when the $\mathrm{pH}$ is lower than 5 or when the concentration of sulfide ion is higher than $0.02 \mathrm{mM}$ was obtained. Through the easy incorporation of biocides into the material and control of the leakage rate, the authors have created an effective and responsive antifouling material with high antibacterial properties against strains of E. Coli.

In 2014, Gorbunova et al. [39] reported water-soluble nanocomposites for disinfection purposes based on silver ions and guanidine-based biocides. The biocides were synthetized through a radical mediated co-polymerization of 2,2-diallyl-1,1,3,3-tetra-ethylguanidiniumchloride with $\mathrm{N}$-vinylpyrrolidone [poly(AGC-VP)] and vinylacetate [poly(AGC-VA)]. To achieve this, aqueous solution containing the previously obtained polymers were treated with $\mathrm{AgNO}_{3}$ under reducing conditions, followed by precipitation of the material. After analysis, the authors report average particle sizes ranging from 52 to $62 \mathrm{~nm}$ for the poly(AGC-VP) and 28 to $30 \mathrm{~nm}$ for the poly(AGC-VA) moieties. Toxicological analysis was conducted in white male mice, and both polymers, together with the corresponding nanocomposites, were found to be non-toxic with LD50 values above the $1000 \mathrm{mg} / \mathrm{kg}$. The antibacterial tests revealed that the nanocomposites were able to provide a broad spectrum of action against several strains of bacteria, including both Gram-positive and Gram-negative bacteria. These materials are based on the action of guanidine-based compounds-a known antibacterial structure-together with the cell membrane disruption properties of silver ions, coupled with the absence of toxicology of these species, being suitable alternatives for the disinfection of surfaces. 
Moya et al. [40] studied the effect of treating tropical wood with silver nanoparticles and the subsequent effect on the white-rot decay disease, caused by a fungus. The silver nanoparticles were synthetized in a simple manner by adding $\mathrm{AgNO}_{3}$ to a solution of $\mathrm{NaBH}_{4}$ and PVP as a stabilizer. The obtained nanoparticles were then inserted, through a pressure device, in fine plaques of three different types of tropical woods native from the Costa Rica flora (Acacia mangium, Cedrela odorata, and Vochysia guatemalensis). The effectiveness of silver nanoparticles in these species was measured by the degradation of wood against fungal attack, specifically resistance to white decay disease caused by Trametes versicolor. In every case, the addition of silver nanoparticles in the wood proved efficient in preventing the action of the fungal decay. When silver nanoparticles were added, weight loss in the wood samples was less than $5 \%$ for both heartwood and sapwood of the three species, as can be observed in Figure 11. These results confirm that the effect was caused by the application of silver nanoparticles as a wood preservative and can lay the ground for the use of such silver nanoparticles in the prevention and conservation of wood. A broader study on the same subject was conducted by the same research team in 2017 [41]. Silver nanoparticles were synthetized in a similar fashion, though in this study, polyethylene glycol was used in excess as the reducing agent. The obtained set of nanoparticles was pressed in wood, using the same protocol already described, though this time, a bigger set of tropical woods was used to broaden the study of silver nanoparticles in the prevention of the white-ort fungal disease. The authors found that different plant species tend to have different retention capacities for the nanoparticle materials, which can be related to the number of nanoparticles absorbed by the wood and the potency of the antibiotic effect. Nonetheless, all the tested trees improved their stability, durability, and resistance to the white-rot fungus T. Versicolor, as it is shown in Figure 12b. However, the silver nanoparticles could not produce any significant effect on the resistance of the brown-rot fungal disease caused by L. Acuta, showcased in Figure 12a.

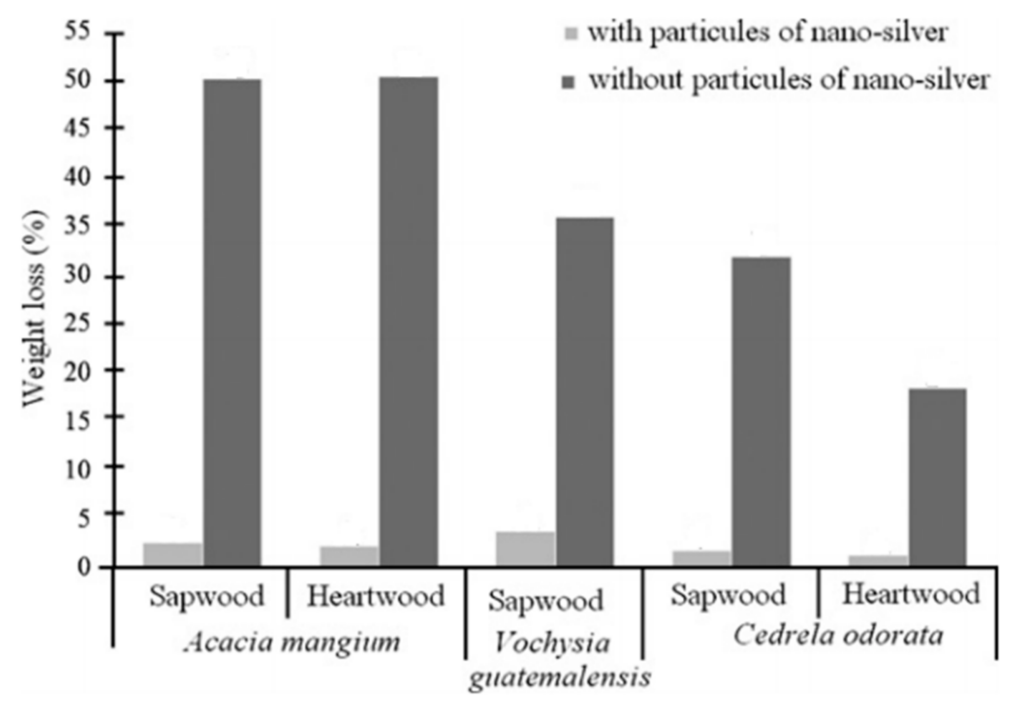

Figure 11. Relative weight loss in the wood samples with and without AgNPs treatment. The weight loss in wood is a condition of the rotting caused by Trametes versicolor. Image retrieved from [40]. 

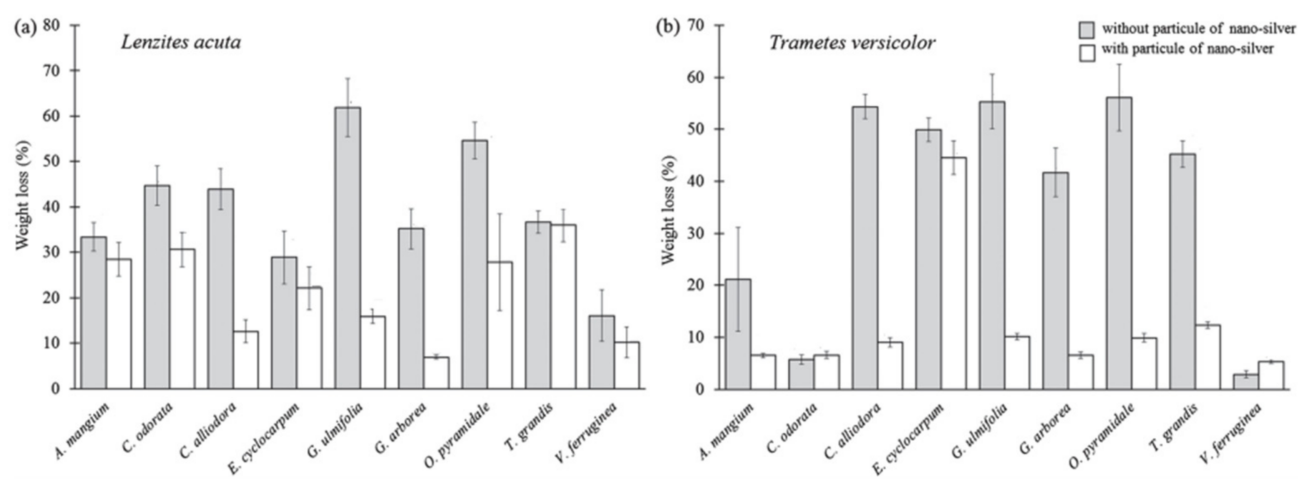

Figure 12. Relative weight loss in the wood samples with and without AgNPs treatment, for the diseases caused by the fungus Lenzites acuta (a) and Trametes versicolor (b), for several different types of wood. Image retrieved from [41].

In 2015, Palza et al. [42] reported the metal release profile of copper nanoparticles embedded in polypropylene matrixes. As copper is already a known antibacterial, the authors studied many variables that may influence the effectiveness of disinfection, such as pre-dispersion of particles in liquid solvents, polymer molecular weight, melt mixing conditions, addition of a compatibilizer agent, and surface functionalization of the metal particles. The polymeric matrix used in this study was a commercial-grade polypropylene that was loaded with a set of commercial Copper-Nanoparticles (CuNPs), with a median particle size of $10 \mathrm{~nm}$. The amount of CuNPs in the composites was determined to be $5 \mathrm{wt} \%$. The original CuNP agglomerates, having an average size of $70 \mu \mathrm{m}$, were drastically reduced to a final average size of $30 \mu \mathrm{m}$ by either pre-dispersing the particles in ethanol or using a compatibilizer. In addition, surface functionalized CuNPs prevent the formation of micrometric agglomerates. This study showed that by tuning the properties of CuNPs, it is possible to increase their antibacterial profile.

Bing et al. [43] prepared photoactive nanohybrids using graphitic carbon nitride (g$\mathrm{C}_{3} \mathrm{~N}_{4}$ ) nanosheets with embedded $\mathrm{Ag}$ nanoparticles $\left(\mathrm{Ag} / \mathrm{g}-\mathrm{C}_{3} \mathrm{~N}_{4}\right)$. The bulk graphitic carbon matrix was synthetized by polymerization of melamine at elevated temperatures, to which it was followed by the addition of silver nitrate under reducing conditions to produce the desired material. This device is driven by visible light irradiation, from which reactive oxygen species (ROS) are formed that damage organic material in bacteria. With visible light irradiation, the production of ROS by the material $\mathrm{Ag} / \mathrm{g}-\mathrm{C}_{3} \mathrm{~N}_{4}$ has increased considerably, when compared with the pristine $\mathrm{g}-\mathrm{C}_{3} \mathrm{~N}_{4}$ nanosheets, and moreover, the nanohybrids showed enhanced antibacterial efficacy and ability to disperse bacterial biofilms. The results demonstrated that after $12 \mathrm{~h}$ culture, for E. coli and S. aureus, the $\mathrm{Ag} / \mathrm{g}-\mathrm{C}_{3} \mathrm{~N}_{4}$ nanohybrids exhibited a strong antibacterial activity. The inactivation efficiency could nearly reach $100 \%$, and $\mathrm{Ag} / \mathrm{g}-\mathrm{C}_{3} \mathrm{~N}_{4}$ were able to effectively inhibit the development of $E$. Coli colonies, and the antibacterial activity was improved upon increasing the time of visible light irradiation. The results suggest that biofilms are subject to a fast and dose-dependent response of the nanohybrids, with the latter being able to destroy up to $70 \%$ of the biofilm mass. The authors proposed that the visible light irradiation of the nanosheets produces band electrons and valence band holes, which act to damage organic material in bacteria. In this model, the embedded AgNPs act as electron traps. The enhanced antibacterial properties and biofilm-disrupting ability of the nanohybrids may offer many biomedical applications as the results demonstrated that the $\mathrm{Ag} / \mathrm{g}-\mathrm{C}_{3} \mathrm{~N}_{4}$ nanohybrids are effective for biofilm elimination and could also cause bacterial death in the residual biofilms.

In 2016, Lee et al. [44] studied the effect of coating the surface of silica nanoparticles with different amine-based polymer molecules. The selected molecules in this study were based on amine functionalities, and were polypyrrole (Ppy), diallylamine, 2-(tert-butylamino)ethylmethacrylate (TBAM), and dimethylaminoethyl methacrylate (DMAEMA), which were then coupled to the surface of the nanoparticles through va- 
por deposition method (VDP). Then, the products were treated with 1-bromohexane to promote the quaternization of the amines. Because it is well established that quaternary ammonium salts are very effective antibacterial agents, the authors used that knowledge to shield the material with antibacterial properties. However, despite its charge, Ppy is not a quaternary ammonium salt and thus it would be expected to behave differently from the remaining materials. In fact, Ppy was not able to produce any considerable effect on the bacteria, which proves the statement postulated by the authors that under moist conditions, the amino group of PPy was not protonated, resulting in an absence of biocidal performance. As for the other nanoparticles, they were all able to kill bacteria within the time scope of one hour, despite all of them having different attacking profiles and different efficiencies, as can be observed in Figure 13.

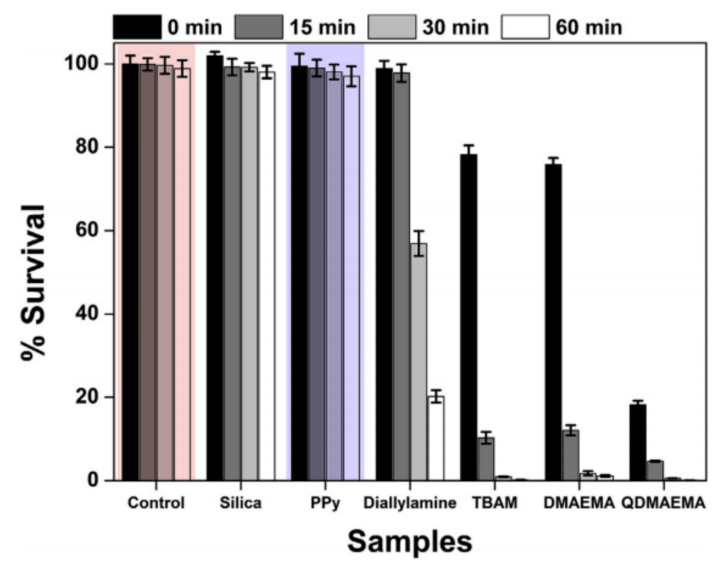

Figure 13. Graphical representation of the relative survival rate of the different NPs functionalized with different quaternary amines. Retrieved from [44].

Gehring et al. [45] designed a system based on mesoporous organosilica (PMOs) nanoparticles that simultaneous releases nitric oxide (NO) in combination with singlet oxygen and superoxide radicals $\left(\mathrm{O}_{2} \bullet^{-}\right)$, as reactive oxygen species (ROS), with significantly enhanced biocidal activity. The pristine nanoparticles were obtained by adapting a Stöber protocol for the synthesis of thiol functionalized PMOs NPs. The synthesis was achieved following a two-step reaction, using 1,5-bis-tri(isopropoxysilyl)-benzene-3-thiol $\left.\left.\left(\mathrm{PrO}^{\mathrm{i}}\right) 3 \mathrm{Si}\right) 2 \mathrm{PhSH}\right)$ as $\mathrm{SH}$ precursor and $\mathrm{CTAB}$ and Brij-56 as surfactants. NPs were posteriorly modified via S-nitrosothiol groups by reaction of the thiol groups with $\mathrm{NaNO}_{2}$, and the ROS-producing photosensitizer Rose Bengal was attached via thiol-ene-click-chemistry (Figure 14). To test the antibacterial effect, the material was irradiated with visible light and assessed for the ability to interfere with bacterial colonies. It was found that the results show a high bactericidal activity and complete destruction of colonies and thus the way is open for the incorporation of this technology in the disinfection control of medical applications, or for other uses, as there exists a huge versatility of possible interactions between the thiol groups and materials such as cotton fibers. 


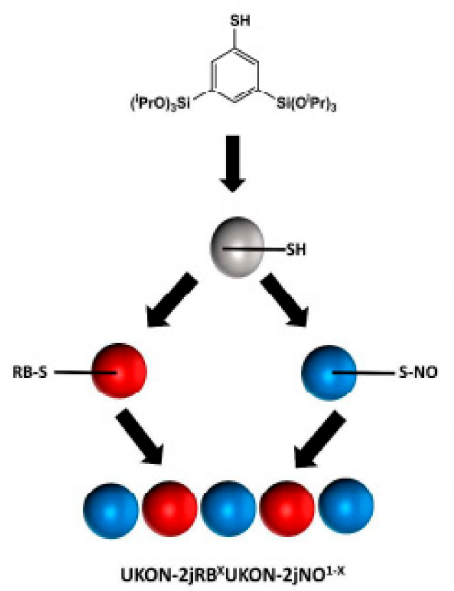

Figure 14. Graphical representation of the relative survival rate of the different NPs functionalized with different quaternary amines. Retrieved from [45].

Procaccini et al. [46] developed an antibacterial coating for surgical-grade stainless steel based on methyl-silica hybrid materials doped with silver. The authors studied a relationship between the structure of the hybrid matrix, biocide behavior, and the aggregation state of silver ions as a function of the densifying thermal treatment used to produce the materials. The sol-gel material was prepared by hydrolytic condensation, in an acidic medium, of tetraethoxysilane (TEOS) and methyl-triethoxysilane (MTES). Then, $\mathrm{AgNO}_{3}$ (as the supplier of $\mathrm{Ag}^{+}$ions) and silica nanoparticles (to give mechanical reinforcement) were added. The material was deposited on $316 \mathrm{~L}$ stainless steel and microscope glass slides by dip-coating method and densified at 50,150 , and $450{ }^{\circ} \mathrm{C}$. The densification temperatures produced different outcomes regarding the antibacterial effectiveness, and with the increase of the densifying temperature employed, a decrease in the antibacterial activity was observed (for the sample treated at $450{ }^{\circ} \mathrm{C}$ is almost neglectable). The increase in the densifying thermal temperature tends to promote a transformation of $\mathrm{Ag}^{+}$ions to $\mathrm{Ag}^{0}$, forming metallic nanoparticles at $150{ }^{\circ} \mathrm{C}$ that convert into $\mathrm{AgO}$ at higher temperatures. This evolution in the oxidation state of silver is accompanied by an agglomeration process of silver nanoparticles, where the smaller nanoparticles degrade at temperatures higher than $150{ }^{\circ} \mathrm{C}$. Nonetheless, despite the apparent effectiveness of the disinfection properties of this material, the authors also raise awareness for the need to execute more profound and detailed studies, regarding the cytotoxicity of such materials, before they can be effectively implemented in the disinfection of stainless-steel surgical grade medical materials.

In 2017, Mattos et al. [47] prepared a biogenic silica derived from Equisetum arvense as a carrier of a natural biocide agent, a bark neem extract cross-linked with polycarboxylic acids. Due to its high potential as a green and natural biocide, the neem extracts were already used to create controlled-release systems by using carbohydrates and biodegradable polymers. However, the authors point a lack of studies concerning the loading of neem compounds in inorganic supports such as the silica nanostructures. In the synthetic procedure used, the neem extract was treated with 1,2,3,4-Butanetetracarboxylic acid (BTCA) and sodium hypophosphite monohydrate (SHPI) to produce ester bonds between silica surface and neem extract, as can be seen in Figure 15. Upon testing the as-synthetized material, it was found that the nanoparticles have higher thermal stability (when compared with the plain nanoparticles) and a high level of biocide loading. Experiments comprising three sequential release profiles revealed total mass release after 20 days in the unloaded neem material, while the neem bark extract loaded in silica support reached a release magnitude of around $70 \%$ after 30 days. The authors also found that when the neem extract is loaded in the support, a four-fold improvement in the stability in aqueous media is observed. The antioxidant capacity of neem compounds released from silica was also evaluated showing scavenging activity over 50\% of DPPH (2,2-diphenyl-1-picrylhydrazyl). The authors did not provide any testing of antibacterial properties, but since neem bark extract has already 
been studied for the antibacterial properties, it is expected that the material also has some potential against disinfections. However, the bioactivity of the material was evaluated against an economically important leafcutter ant species and revealed a longer time to cause $100 \%$ of mortality than the non-supported biocide, which can be considered as an advantage for practical applications.

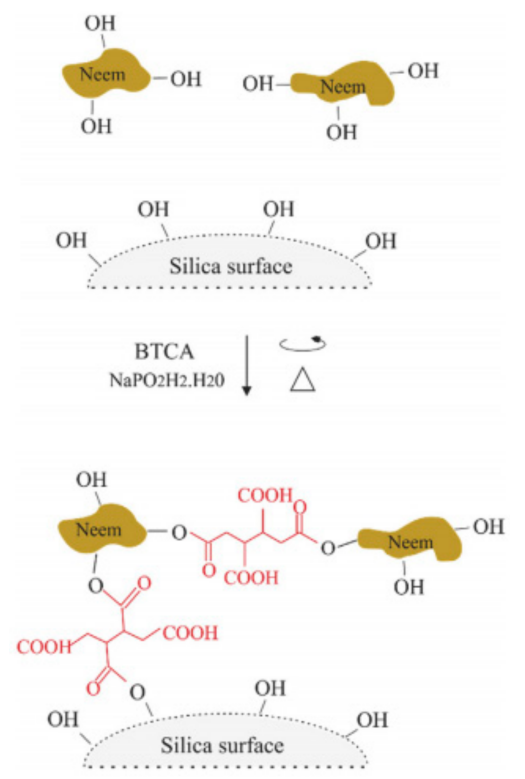

Figure 15. Representation of the method employed for the synthesis of cross-linked Neem Bark Extracts in silica surface with BTCA. Retrieved from [47].

Chan et al. [48] incorporated allyl isothiocyanate (AIT) and cinnamaldehyde (CNAD), as volatile biocides, in mesoporous silica nanoparticles (MSNs) to improve their biocidal activity. Since volatile biocides have the tendency to boil away from the desired surface, their incorporation in nanoparticles could hamper their propensity to disperse and keep the surface resistant to bacteria for longer periods of time. With that in mind, the team synthetized silica nanoparticles with hexagonally ordered mesopores, and loaded them with AIT and CNAD via diffusion in solution. To maximize the loading efficiency, the diffusion was executed with an excess of oil. AIT and CNAD are adsorbed onto the silica surface and in the nanoparticles' pores by hydrogen bonds and van der Waals forces that can be broken by water, which enables them to be released in situ. The loading capacity of the MSNPs was determined to be approximately $7.4 \mathrm{mg}$ for AIT and approximately $0.95 \mathrm{mg}$ for CNAD for every $10 \mathrm{mg}$ MSNPs. Both biocides, when tested against bacterial lines, showed increased antimicrobial activity against Escherichia coli in planktonic form, when packaged into MSNs. Furthermore, the biocide-loaded nanoparticles showed activity against Pseudomonas aeruginosa biofilms, which have inherent resistance to antimicrobial agents. Finally, the authors make a remark on the possibility to expand the method to include other traditional biocides or less-conventional antibacterial compounds to increase the effectiveness and mode of action.

In 2017, Bhardwaj et al. [49] designed a novel way to incorporate silver nanoparticles (SNPs) directly onto cotton fibers through a microwave-assisted protocol. Pre-washed cotton fibers were submerged in a $2.5 \mathrm{M}$ aqueous solution of poly-(diallyldimethylammonium chloride) (PDDA) for $12 \mathrm{~h}$ and then were dried at room temperature. Following these step, the fibers get shielded with positive charges in their surface. The pre-treated fibers were then submerged in a $2 \mathrm{M}$ solution of sodium citrate with the subsequent addition of an aqueous silver nitrate solution and irradiated with microwave radiation. Under such conditions, SNPs are formed directly in the surface of the cotton fiber, coupled to the positive charges given by the PDDA reagent. The authors optimized the protocol by varying the irradiation time and the microwave power, to control the size of SNPs and 
total density of silver atoms. These parameters increase by increasing the microwave power from $100 \mathrm{~W}$ to $600 \mathrm{~W}$. A full representation of the method, from starting materials to the antibacterial testing, is reported in Figure 16. The cotton fibers were tested to assess their antibacterial properties and revealed higher activity against Gram-negative bacteria, such as Salmonella typhimurium, than Gram-positive bacteria, such as Staphylococcus aureus. On the other hand, it was noted that an excellent deactivation of bacterial colonies happened with Escherichia Coli, which suggests the protocol can be effectively employed for the treatment of cotton fibers in decontamination purposes.

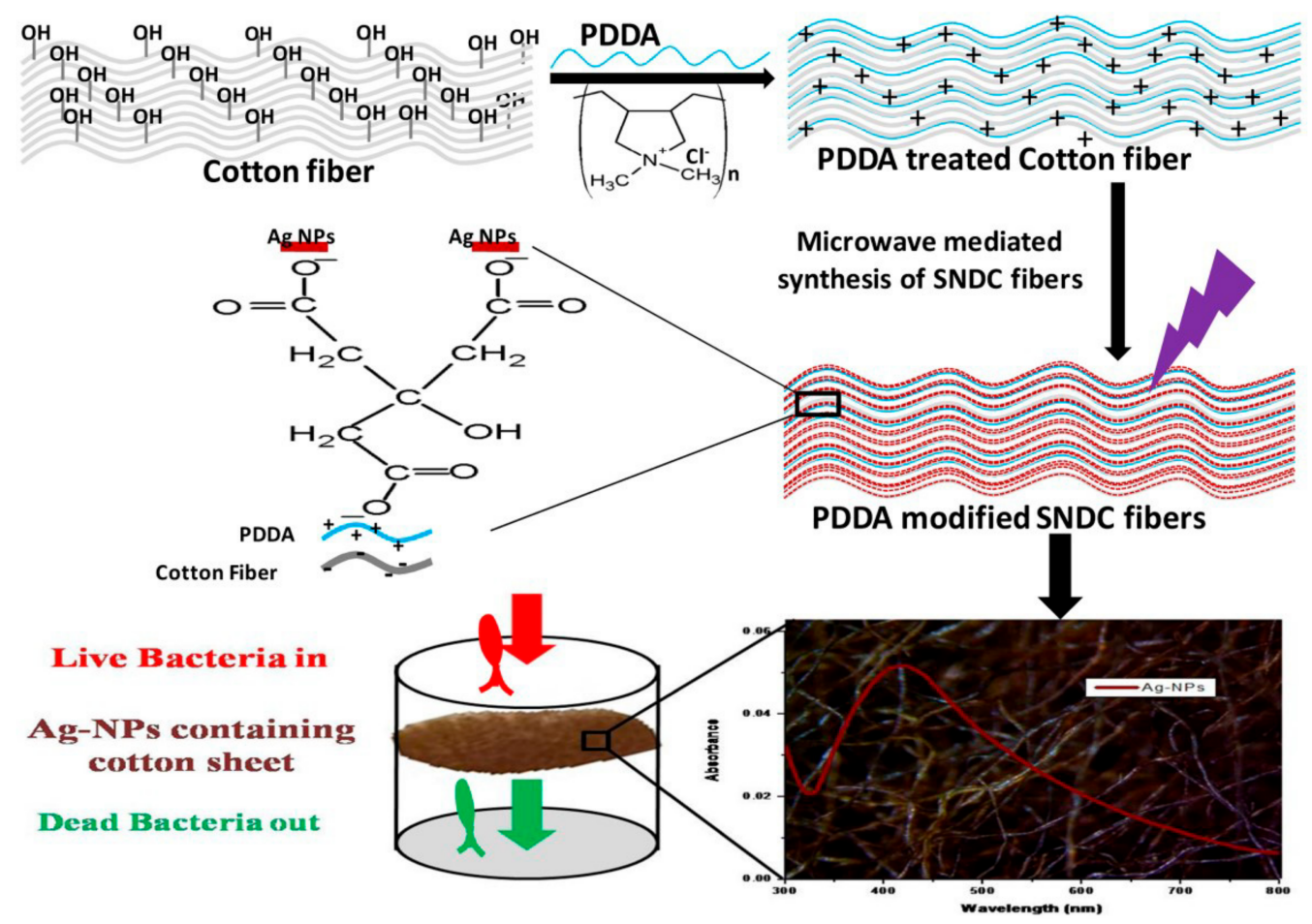

Figure 16. Diagram representing the entire protocol developed by Bhardwaj et al. Retrieved from [49].

Kalaiyarasan et al. [50] reported a simple and fast way to obtain silver nanoparticles loaded with natural biocides from Seabuckethorn (SBT) extracts. These natural biocides have already been extensively reported in the literature [51], as they hold much promise due to their inherent effect against bacteria and for their ability to treat several diseases, such as human cancer. For the preparation of the nanoparticles, a small piece of Whatmann no. 1 filter paper was submerged in a $\mathrm{AgNO}_{3}$ solution for $30 \mathrm{~min}$ and rinsed with water, to remove excess $\mathrm{AgNO}_{3}$. Then, the piece of paper is submerged in a solution containing an aqueous solution of Seabuckethorn extracts for 20 min followed by water rinsing and desiccation. Through this protocol, it was possible to prepare the silver nanoparticles encapsulated with the biocides and supported in a paper matrix (SBT@AgNPs). The antibacterial properties of SBT@AgNPs were evaluated and the results showed high inhibition of Grampositive and Gram-negative bacterial strains, with antibacterial efficacy above 99\% even after one year of storage. The antioxidant properties were also studied and SBT@AgNPs showed good DPPH radical scavenging. Overall, the Seabuckethorn biocides had a better antibacterial and antioxidant activity when encapsulated in SBT@AgNPs. The authors claim the produced material is very stable and highly antiseptic with higher antibacterial efficacy than the two commercially available medicated dressing materials. In addition, SBT@AgNPs can be easily prepared by a low-cost method with high reproducibility and stability, being possible to adapt it to a variety of different materials and surfaces.

In 2019, Haider et al. [52] proposed a single-pot protocol to prepare silica nanoparticles with silver at core and crust (SiNP-AgCC) around a previously produced silver nanoparticle template. The silver nanoparticles were prepared with $\mathrm{AgNO}_{3}$, produced with average 
diameters between 10-20 nm, followed by the addition of TEOS, through a Stöber modified method. In this way, the silica matrix from the condensation of TEOS is formed around the silver nanoparticles. Afterwards, formalin is added to the reaction, which acts to reduce and deposit the remaining silver ions in solution on the surface of the silica matrix. Through this method, it is possible to selectively reduce only the silver ions on the surface because the silica scaffold prevents the leakage of silver from the inside. The silver nanoparticles formed at the surface had average diameters between $2-5 \mathrm{~nm}$. The rate of silver release was studied through a dialysis system and the authors concluded that the material can continuously release silver ions in solution for up to 20 days. The antibacterial tests demonstrated that the material is very active against bacterial colonies, as the colonies were rapidly destroyed upon first contact with SiNP-AgCC. The persistence of such a material is also noteworthy as it was still able to present bactericidal properties even at lower concentrations. Due to the biocompatibility of the material, as well as the ease in preparation, the authors proposed that SiNP-AgCC can be applied in polymer nanocomposite water treatment membranes and also for biomedical uses.

In 2020, Labena et al. [53] prepared different metal nanoparticles capped by a newly synthesized cationic surfactant (CS) to use as biocides and corrosion inhibitors. They designed a system comprising the synthesis of different metal nanoparticles with a cationic surfactant coupled to the surface. The metal NPs (zinc, ZnNPs-C-CS; manganese, MnNPsC-CS and tin, SnNPs-C-CS) were prepared via reduction reactions of metal chlorides by $\mathrm{NaBH}_{4}$ in a solution of the CS. Through this method, the nanoparticle is formed directly with the cationic surfactant attached to the surface. The prepared metal NPs showed higher anti-microbial activities against Gram-negative and Gram-positive bacteria and yeast strains, and higher anti-biofilm activity against aerobic developed bacterial films, than the CS, and the activities were reported in the order of SnNPs-C-CS > MnNPs-C-CS $>$ ZnNPs-C-CS $>$ CS. The authors postulate that this order may be related to the smaller size obtained for SnNPs-C-CS, when compared to the others. Because of its small size, the strontium nanoparticles can more easily penetrate the membrane of bacteria and kill them. The corrosion inhibition properties of metal NPs were also tested, and the results showed an inhibition efficacy above $87 \%$, while the surfactant alone had an efficiency of $85 \%$. These results show that the surface binding of the surfactant was not able to significantly improve the corrosion capacity of itself. On the other hand, the results and versatility of applications of these metal NPs makes them attractive alternatives for the disinfection of several systems and materials.

Presentato et al. [54] developed a controlled release system (CRS) based on mesoporous silica nanoparticles (MSNs) loaded with a biocide for the preservation and conservation of archeologically interesting stone. To achieve this, the team prepared the MSNs by an emulsion-condensation method, which consists in the hydrolysis of alkoxysilanes followed by a reaction of poly-condensation of the resulting silanol molecules around a CTAB surfactant template. Spherical MSNs with average diameters of $55 \mathrm{~nm}$ and pore sizes ranging 3-8 $\mathrm{nm}$ in diameter were obtained. The loading of the biocide was achieved through a method previously developed by the same team [55], which involves soaking the MSNs powder in a solution of known concentration of the biocide. The active compound encapsulated in MSNs was Preventol RI-80, a known preservative used in the conservation of archaeological stone. The rate of biocide released from the material was studied in stone disks and the antibacterial effect was also monitored. The authors found that the stone treated with the material was able to resist bacterial blooming for up to 12 months, while at the same time, the material exhibited high bactericidal properties, even greater than other known stone conservatives, used as the control. These results show the material synthetized is effective in slowly and steadily releasing the biocide in the desired site of action. Overall, the authors were successful in demonstrating the usefulness of the material in the preservation of stone. Furthermore, the encapsulation of the biocide in MSNs increased the strength of its action, which opens the way for the encapsulation of other known stone preservatives for the purposes of protecting archaeological materials. 
Lara et al. [56] prepared silver nanoparticles (AgNPs, with average diameters ranging from 1-3 nm) by a microwave-assisted approach and tested their activity against the biofilms formed by strains of Candida Auris, which is regarded as a prominent pathogenic fungus that can survive for several days attached to different surfaces. The antibiotic tests against $C$. Auris revealed a potent inhibitory capacity against the fungus, the formation of biofilms, and even against pre-formed biofilms. A detailed imaging analysis showed that the nanoparticles were able to cause cell wall damage on the microorganisms and subsequently kill them. The authors treated several medical surfaces with AgNPs and found very positive conclusions regarding the prospects of surface disinfection. Silicone elastomers and bandage dressings treated with the material showed an efficient preventive action against the formation and contamination by the bacterial biofilms. Furthermore, the same materials were thoroughly washed several times with a phosphate buffer solution and still were able to maintain the fungicidal action against $C$. Auris for up to six cycles of washing. The material proposed by the authors is effective in the preventive treatment of medical-based surfaces.

In conclusion, over recent years, several efforts have been made towards the development of materials capable of exclusive and directed disinfection mechanisms. It is clear that there is a trend towards the delivery of encapsulated biocides in nanomaterials for slow-release or controlled disinfection purposes, which has the ability to allow for new strategies in surface cleaning and prevention of bacteria biofilm formation.

The biocidal action of silver ions stands out more than the remainder of other metal ions or non-metal biocides. The ease of encapsulation or integration of the metallic silver ions (either through Stöber processes or through controlled redox reactions) on the nanomaterial core, shell, or interface allowed for a diversity of new materials and strategies to be developed. In most of the cases, the use of silver ions in under-toxicity levels permitted a control disinfection of the material and, in some cases, it even addressed the biofilm formation distress.

The encapsulation of other biocides, with particular emphasis on naturally occurring biocides, was also demonstrated throughout this review with varying results. The versatility of the obtained materials may open the path towards an unlimited range of different possibilities, allowing the tuning of several different biocides, and its respective concentration, as well as the rate of release and the adherence to the desired surface. Furthermore, the studies pin-pointed here are extremely diverse in nature, whether they regard the biocidal agent used or the material on which it acted. It is possible to infer that, with such great and relevant work reported in the area over the last few years, the use of nanomaterials for disinfection purposes may hold great promise for the future.

\section{Conclusions}

Efflux mechanisms are known to be important in antibiotic resistance, but it is questionable whether the observed increased MICs of biocides could have clinical implications for hard-surface or topical disinfection $(423,428)$. It has been speculated that low-level resistance may aid in the survival of microorganisms at residual levels of antiseptics and disinfectants; any possible clinical significance of this remains to be tested. With growing concerns about the development of biocide resistance and cross-resistance with antibiotics, it is clear that clinical isolates should be under continual surveillance and possible mechanisms should be investigated.

It is clear that antiseptic and disinfectant compounds can be designed in order to increase anti-microbial and active surface activities. It is important to note that some particular antiseptic or disinfectant compounds are selected according to circumstances or nosocomial outbreaks; for example, certain active agents are clearly more efficacious against Gram-positive than Gram-negative bacteria. Few reports have further investigated increased bactericidal concentrations or actually used dilutions of products, which in many cases contain significantly higher concentrations of biocides, or formulation attributes, which can increase product efficacy; in a number of cases, changes in the MICs have 
actually been shown not to be significant. Efflux mechanisms are known to be important in antibiotic resistance, and the increased MICs of biocides could have clinical implications for hard-surface or topical disinfection.

This review is focused on two alternative classes of antiseptic and disinfectants based on:

(1) Ionic systems such as ionic surfactants (cationic and anionic) and ionic liquids;

(2) Nanomaterials and nanoparticles.

In conclusion, several organic salts (Ionic Systems) showed great potential for application in different kinds of surfaces. Although significant progress has been made with bacterial investigations, a greater understanding of these mechanisms is clearly lacking for other infectious agents. Studies of the mechanisms of action of and microbial resistance to antiseptics and disinfectants is very important in this field.

Alternatively, silica or metal nanoparticles can be relevant for application as an antiseptic or disinfectant, producing good performances in different surfaces. Other nanomaterials can be tested with improving antimicrobial activities.

The big challenge in the development of efficient antiseptic and disinfectant products can involve new ionic systems as well as nanomaterials. The combination of the surfactant salts with nanomaterials or nanoparticles seems to be promising as more effective active surface agents as antiseptics and disinfectants.

Author Contributions: Conceptualization, Z.P., M.M.S., S.G., and L.C.B.; writing-original draft preparation, F.F. and L.F.; writing-review and editing, L.C.B. and S.G.; supervision, L.C.B. and S.G.; funding acquisition, L.C.B. All authors have read and agreed to the published version of the manuscript.

Funding: The authors give thanks to Associate Laboratory for Green Chemistry-LAQV, which is financed by national funds from FCT/MCTES (UIDB/50006/2020) and co-financed by the ERDF under the PT2020 Partnership Agreement (POCI-01-0145-FEDER-007265). We also thanks to FCT (PTDC/QUI-QOR/32406/2017), MAR2020 (MAR-02.01.01-FEAMP-0042-INOVA4AQUA) projects and Solchemar company.

Institutional Review Board Statement: Not applicable.

Informed Consent Statement: Not applicable.

Conflicts of Interest: The authors declare no conflict of interest.

\section{References}

1. McDonnell, G.; Russell , A.D. Antiseptics and Disinfectants: Activity, Action, and Resistance. Clin. Microbiol. Rev. 1999, 12, 147-179. [CrossRef] [PubMed]

2. Saravanan, A.; Kumar, P.S.; Hemavathy, R.V.; Jeevanantham, S.; Kamalesh, R.; Sneha, S.; Yaashikaa, P.R. Methods of detection of food-borne pathogens: A review. Environ. Chem. Lett. 2021, 19, 189-207. [CrossRef]

3. Hosoon Choi, P.C.; Lichtfouse, E.; Martel, J.A.; Hwang, M.; Jinadatha, C.; Sharma, V.K. Classical and alternative disinfection strategies to control the COVID-19 virus in healthcare facilities: A review. Environ. Chem. Lett. 2021, in press.

4. Schick, M.J.; Polymers, T.F. Tadros Editor; Academic: Orlando, FL, USA, 1985.

5. Rosen, M. Surfactants and Interfacial Phenomena. In Surfactants and Interfacial Phenomena; Wiley-VCH: Hoboken, NJ, USA, 2004.

6. Shinoda, K. Solvent Properties of Surfactant Solutions. Soil Sci. 1968, 4. [CrossRef]

7. Dave, N. A Concise Review on Surfactants and Its Sigificance. Int. J. Appl. Chem. 2017, 3, 663-672.

8. Lei, Z.; Koo, C.B.; Macfarlane, Y.M.D.R. Introduction: Ionic Liquids. Chem. Rev. 2017, 10, 6633-6635. [CrossRef]

9. Pendleton, J.N.; Gilmore, B.F. The antimicrobial potential of ionic liquids: A source of chemical diversity for infection and biofilm control. Int. J. Antimicrob. Agents 2015, 46, 131-139. [CrossRef]

10. Klembt, S.; Eckstein, M.; Kragl, U. Biocatalytic Reactions in Ionic Liquids. In Ionic Liquids in Synthesis; Peter Wasserscheid, T.W., Ed.; Wiley Online Library: Hoboken, NJ, USA, 2007.

11. Blesic, M.; Plechkova, M.M.; Seddon, N.V.; Rebelo, L.P.N.; Lopes, A. Sef-aggregation of ionic liquids: Micelle formation in aqueous solution. Green Chem. 2007, 5, 481-549. [CrossRef]

12. Pernak, J.; Lota, G.; Frackowiak, E. Synthesis and properties of trigeminal tricationic ionic liquids. Chem. A Eur. J. 2007, 11, 3106-3112. [CrossRef]

13. Luczak, J.J.C.; Lacka, I.; Stolte, S.; Hupka, J. Antimicrobial and surface activity of 1-Alkyl-3-Methylimidazolium Derivatives. Green Chem. 2010, 12, 593. [CrossRef] 
14. Jungnickel, C.Ł.J.; Ranke, J.; Fernández, J.F.; Müller, A.; Thöming, J. Micelle formation of imidazolium ionic liquids in aqueous solution. Colloid. Surf. A 2008, 1, 278-284. [CrossRef]

15. Samori, C. Ionic Liquids and their Biological Effects towards Microorganisms. Curr. Org. Chem. 2012, 12, 1888-1904. [CrossRef]

16. Pernak, J.S.A.; Bogacki, M.B. Quantitative relation between surface active properties and antibiotic activity of 1-alkyl-3alkylthiomethylimidazolium chlorides. Curr. Org. Chem. 1995, 11, 2019-2020. [CrossRef]

17. Pernak, J.S.A. 3-Alkylthiomethyl-1-ethylimidazolium chlorides. Correlation between critical micelle concentra-tions and minimum inhibitory concentrations. Eur. J. Med. Chem. 1996, 11, 901-903. [CrossRef]

18. Demberelnyamba, D.K.K.-S.; Choi, S.; Park, S.-Y.; Lee, H.; Kim, C.-J.; Yoo, I.-D. Synthesis and antimicrobial proper-ties of imidazolium and pyrrolidinonium salts. Bioorg. Med. Chem. 2004, 5, 853-857. [CrossRef]

19. Pernak, J.; Feder-Kubis, J.; Cieniecka-Rosłonkiewicz, A.; Fischmeister, C.; Griffin, S.T.; Rogers, R.D. Synthesis and properties of chiral imidazolium ionic liquids with a (1R,2S,5R)-(-)-menthoxymethyl substituent. New J. Chem. 2007, 31, 879-892. [CrossRef]

20. Foksowicz-Flaczyk, J.; Walentowska, J. Eco-Friendly Antimicrobial Finishing of Natural Fibres. Mol. Cryst. Liq. Cryst. 2008, 484, 207-212. [CrossRef]

21. Cybulski, J.W.A.; Kulig-Adamiak, A.; Da̧browski, Z.; Praczyk, T.; Michalczyk, A.; Walkiewicz, F.; Materna, K.; Pernak, J. Mandelate and prolinate ionic liquids: Synthesis, characterization, catalytic and biological activity. Tetrahedron Lett. 2011, 12, 1325-1328. [CrossRef]

22. Yagci, M.; Bolca, S.; Heuts, H.; Ming, W.; de With, G. Antimicrobial polyurethane coatings based on ionic liquid quaternary ammonium compounds. Prog. Org. Coat. 2011, 72, 343-347. [CrossRef]

23. Venkata Nancharaiah, Y.; Kumar Reddy, G.K.; Lalithamanasa, P.; Venugopalan, V.P. The ionic liquid 1-alkyl-3-methylimidazolium demonstrates comparable anti-microbial and antibiofilm behavior to a cationic surfactant. Biofouling 2012, 28, 1141-1149. [CrossRef]

24. El-Shamy, A.M.; Zakaria, K.; Abbas, M.A.; Zein El Abedin, S. Anti-bacterial and anti-corrosion effects of the ionic liquid 1-butyl-1-methylpyrrolidinium trifluoromethylsulfonate. J. Molec. Liquid. 2015, 211, 363-369. [CrossRef]

25. Singh, V.; Tyagi, R. Physicochemical properties and effect of organic and inorganic electrolytes on surface properties of C12 and C16 alcohol-based bis-sulfosuccinate anionic gemini surfactants. Colloid Polym. Sci. 2017, 295, 601-611. [CrossRef]

26. Marek, J.; Joskova, V.; Dolezal, R.; Soukup, O.; Benkova, M.; Fucikova, A.; Malinak, D.; Bostikova, V.; Kuca, K. Synthesis, Antimicrobial Effect and Surface Properties of Hydroxymethylsubstituted Pyridini-um Salts. Lett. Drug Design Discov. 2018, 15, 828-842. [CrossRef]

27. Ossowicz, P.J.E.; Błaszak, M.; Zatoń, K.; Rozwadowski, Z. Benzalkonium salts of amino acids—Physicochemical properties and anti-microbial activity. Tenside Surfactant. Deterg. 2017, 6, 500-509. [CrossRef]

28. Forero-Doria, O.; Araya-Maturana, R.; Barrientos-Retamal, A.; Morales-Quintana, L.; Guzmán, L. N-alkylimidazolium Salts Functionalized with p-Coumaric and Cinnamic Acid: A Study of Their Antimicrobial and Antibiofilm Effects. Molecules 2019, 24, 3484. [CrossRef]

29. Rzemieniecki, T.G.D.; Rybak, K.; Materna, K.; Juś, K.; Pernak, J. Synthesis, Properties, and Antimicrobial Activi-ty of 1-Alkyl-4hydroxy-1-methylpiperidinium Ionic Liquids with Mandelate Anion. ACS Sustain. Chem. Eng. 2019, 17, 15053-15063. [CrossRef]

30. Anandkumar, B.; George, R.P.; Philip, J. Efficacy of imidazolium and piperidinium based ionic liquids on inhibiting bio-film formation on titanium and carbon steel surfaces. Anal. Chim. Acta 2020, 1126, 38-51. [CrossRef]

31. Smith, C.A.; Cataldo, V.A.; Dimke, T.; Stephan, I.; Guterman, R. Antibacterial and Degradable Thioimidazolium Poly(ionic liquid). ACS Sustain. Chem. Eng. 2020, 8, 8419-8424. [CrossRef]

32. Sawada, H.; Kakehi, H.; Koizumi, M.; Katoh, Y.; Miura, M. Preparation and antibacterial activity of novel fluoroalkyl end-capped oligomers/silica nano-composites-encapsulated low molecular biocides. J. Mater. Sci. 2007, 42, 7147-7153. [CrossRef]

33. Ghosh, S.; Yadav, S.; Vasanthan, N.; Sekosan, G. A study of antimicrobial property of textile fabric treated with modified dendrimers. J. Appl. Polym. Sci. 2010, 115, 716-722. [CrossRef]

34. Chapman, J.; Weir, E.; Regan, F. Period four metal nanoparticles on the inhibition of biofouling. Colloids Surfaces B Biointerfaces 2010, 78, 208-216. [CrossRef] [PubMed]

35. Miranda, M.; Fernández, M.M.; Lopez-Esteban, S.; Malpartida, F.; Moya, J.S.; Torrecillas, R. Ceramic/metal biocidal nanocomposites for bone-related applications. J. Mater. Sci. Mater. Med. 2012, 23, 1655-1662. [CrossRef] [PubMed]

36. Das, S.K.; Khan, M.R.; Parandhaman, T.; Laffir, F.; Guha, A.K.; Sekaran, G.; Mandal, A.B. Nano-silica fabricated with silver nanoparticles: Antifouling adsorbent for efficient dye removal, effective water disinfection and biofouling control. Nanoscale 2013, 5, 5549-5560. [CrossRef] [PubMed]

37. Liu, H.; Lee, Y.-Y.; Norsten, T.B.; Chong, K. In situ formation of anti-bacterial silver nanoparticles on cotton textiles. J. Ind. Text. 2014, 44, 198-210. [CrossRef]

38. Zheng, Z.; Huang, X.; Schenderlein, M.; Borisova, D.; Cao, R.; Möhwald, H.; Shchukin, D.; Moehwald, H. Self-Healing and Antifouling Multifunctional Coatings Based on $\mathrm{pH}$ and Sulfide Ion Sensitive Nanocontainers. Adv. Funct. Mater. 2013, 23, 3307-3314. [CrossRef]

39. Marina Gorbunova, L.L. New biocide guanidine-containing nanocomposites. J. Nanopartic. Res. 2014, 16, 1-9. [CrossRef]

40. Róger Moya, A.B.; Rodriguez-Zuñiga, A.; Vega-Baudrit, J. Effect of silver nanoparticles on white-rot wood decay and some physical properties of three tropical wood species. Wood Fiber Sci. 2014, 4, 527-538. 
41. Moya, R.; Rodriguez-Zuniga, A.; Alexander, B.; Vega-Baudrit, J. Effect of Silver Nanoparticles Synthesized with NPsAg-Ethylene Glycol (C2H6O2) on Brown Decay and White Decay Fungi of Nine Tropical Woods. J. Nanosci. Nanotechnol. 2017, 17, 5233-5240. [CrossRef]

42. Palza, H.; Delgado, K.; Pinochet, I. Improving the metal ion release from nanoparticles embedded in a poly-propylene matrix for antimicrobial applications. J. Appl. Polym. Sci. 2015, 132. [CrossRef]

43. Bing, W.; Chen, Z.; Sun, H.; Shi, P.; Gao, N.; Ren, J.; Qu, X. Visible-light-driven enhanced antibacterial and biofilm elimination activity of graphitic carbon nitride by embedded Ag nanoparticles. Nano Res. 2015, 8, 1648-1658. [CrossRef]

44. Lee, I.; Roh, J.; Lee, J.; Song, J.; Jang, J. Antibacterial performance of various amine functional polymers coated silica nanoparticles. Polymers 2016, 83, 223-229. [CrossRef]

45. Gehring, J.; Trepka, B.; Klinkenberg, N.; Bronner, H.; Schleheck, D.; Polarz, S. Sunlight-Triggered Nanoparticle Synergy: Teamwork of Reactive Oxygen Species and Nitric Oxide Released from Mesoporous Organosilica with Advanced Antibacterial Activity. J. Am. Chem. Soc. 2016, 138, 3076-3084. [CrossRef]

46. Procaccini, R.; Bouchet, A.; Pastore, J.; Studdert, C.; Ceré, S.; Pellice, S. Silver-functionalized methyl-silica hybrid materials as antibacterial coatings on surgical-grade stainless steel. Prog. Org. Coat. 2016, 97, 28-36. [CrossRef]

47. Mattos, B.D.; Rojas, O.; Magalhães, W.L. Biogenic silica nanoparticles loaded with neem bark extract as green, slow-release biocide. J. Clean. Prod. 2017, 142, 4206-4213. [CrossRef]

48. Chan, A.C.; Cadena, M.B.; Townley, H.E.; Fricker, M.D.; Thompson, I.P. Effective delivery of volatile biocides employing mesoporous silicates for treating biofilms. J. R. Soc. Interface 2017, 14, 20160650. [CrossRef]

49. Bhardwaj, A.K.; Shukla, A.; Mishra, R.K.; Singh, S.; Mishra, V.; Uttam, K.N.; Singh, M.P.; Sharma, S.; Gopal, R. Power and Time Dependent Microwave Assisted Fabrication of Silver Nanoparticles Decorated Cotton (SNDC) Fibers for Bacterial Decontamination. Front. Microbiol. 2017, 8, 330. [CrossRef]

50. Kalaiyarasan, T.; Bharti, V.K.; Chaurasia, O.P. One pot green preparation of Seabuckthorn silver nanopar-ticles (SBT@AgNPs) featuring high stability and longevity, antibacterial, antioxidant potential: A nano disinfect-ant future perspective. RSC Adv. 2017, 7, 51130-51141. [CrossRef]

51. Upadhyay, N.K.; Kumar, M.Y.; Gupta, A. Antioxidant, cytoprotective and antibacterial effects of Sea buckthorn (Hippophae rhamnoides L.) leaves. Food Chem. Toxicol. 2010, 48, 3443-3448. [CrossRef]

52. Haider, M.S.; Shao, G.; Ahmad, A.; Imran, S.M.; Abbas, N.; Abbas, G.; Hussain, M.; Kim, H.T. Facile, single-pot preparation of nanoporous $\mathrm{SiO} 2$ particles (carrier) with AgNPs at core and crust for controlled disinfectant release. J. Saudi Chem. Soc. 2019, 23, 828-835. [CrossRef]

53. Labena, A.; Hegazy, M.A.; Kamel, W.M.; Elkelish, A.; Hozzein, W.N. Enhancement of A Cationic Surfactant by Capping Nanoparticles: Synthesis, Characterization and Multiple Applications. Molecules 2020, 25, 2007. [CrossRef]

54. Presentato, A.; Armetta, F.; Spinella, A.; Martino, D.F.C.; Alduina, R.; Saladino, M.L. Formulation of Mesoporous Silica Nanoparticles for Controlled Release of Antimicrobials for Stone Preventive Conservation. Front. Chem. 2020, 8, 8. [CrossRef] [PubMed]

55. Saladino, M.L.; Rubino, S.; Colomba, P.; Girasolo, M.A.; Chillura Martino, D.F.; Demirbag, C.; Caponetti, E. Pt(II) complex @mesoporous silica: Preparation, characterization and study of release. Biointerf. Res. Appl. Chem. 2016, 6, 1621-1626.

56. Humberto, H.; Lara, L.I.-T.; Yacaman, M.J.; Lopez-Ribot, J. Inhibition of Candida auris Biofilm For-mation on Medical and Environmental Surfaces by Silver Nanoparticles. ACS Appl. Mater. Interf. 2020, 12, 21183-21191. 